\title{
Spherical Harmonic Solution of the Robin Problem for the Helmholtz Equation in a Supershaped Shell*
}

\author{
Diego Caratelli ${ }^{1}$, Johan Gielis ${ }^{2}$, Ilia Tavkhelidze ${ }^{3}$, Paolo Emilio Ricci ${ }^{4}$ \\ ${ }^{1}$ Microwave Sensing, Signals and Systems, Delft University of Technology, Delft, The Netherlands \\ ${ }^{2}$ Department of Bioscience Engineering, University of Antwerp, Antwerp, Belgium \\ ${ }^{3}$ Faculty of Exact and Natural Sciences, Tbilisi State University, Tbilisi, Georgia \\ ${ }^{4}$ Faculty of Engineering, Campus Bio-Medico University, Rome, Italy \\ Email: d.caratelli@tudelft.nl
}

Received August 11, 2012; revised September 11, 2012; accepted September 19, 2012

\begin{abstract}
The Robin problem for the Helmholtz equation in normal-polar shells is addressed by using a suitable spherical harmonic expansion technique. Attention is in particular focused on the wide class of domains whose boundaries are defined by a generalized version of the so-called "superformula" introduced by Gielis. A dedicated numerical procedure based on the computer algebra system Mathematica ${ }^{\circ}$ is developed in order to validate the proposed methodology. In this way, highly accurate approximations of the solution, featuring properties similar to the classical ones, are obtained.
\end{abstract}

Keywords: Robin Problem; Helmholtz Equation; Spherical Harmonic Expansion; Gielis Formula; Supershaped Shell

\section{Introduction}

Many problems of mathematical physics and electromagnetics are related to the Laplacian differential operator. Among them, it is worth mentioning those relevant to the Laplace and Helmholtz equations. However, most of the mentioned differential problems can be solved in explicit way only in canonical domains with special symmetries, such as intervals, cylinders or spheres [1]. The solution in more general domains can be obtained by using the Riemann theorem on conformal mappings and the relevant invariance of the Laplacian [2]. However, it is clear that conformal mapping techniques can not be used in the three-dimensional case where approaches based on suitable spatial discretization procedures, such as such as finite-difference or finite-element methods, are usually adopted [3].

Different techniques have been proposed in order to solve the mentioned class of differential problems both from a theoretical and numerical point of view (e.g., representing the solution by means of boundary layer techniques [4], solving the corresponding boundary integral equation by iterative methods [5], approximating the relevant Green function by means of the least squares fitting technique [6], solving the linear system relevant to an elliptic partial differential equation by means of relaxation methods [7]). However, none of the contribu-

${ }^{*}$ This research has been partly carried out under the grant PRIN/2006 Cap. 7320. tions already available in the scientific literature deals with the classical Fourier projection method [8] which has been extended in recent papers [9-16] in order to address boundary-value problems (BVPs) in simply connected starlike domains whose boundaries may be regarded as an anisotropically stretched unit circle or sphere centered at the origin.

In this contribution, a suitable methodology, based on the theory of spherical harmonics [17], has been developed in order to compute the solution of the Robin problem for the Helmholtz equation in normal-polar shell-like domains. In particular, the boundaries of the considered domains are supposed to be defined by a generalized version of the so-called Gielis formula (also known as "superformula") [18]. Regular functions are assumed to describe the boundary values, but the proposed approach can be easily generalized in case of weakened hypotheses.

In order to verify and validate the developed technique, a suitable numerical procedure based on the computer algebra system Mathematica ${ }^{\odot}$ has been adopted. By using such procedure, a point-wise convergence of the spherical harmonic series representation of the solution has been observed in the regular points of the boundaries, with Gibbs-like phenomena potentially occurring in the quasi-cusped points. The obtained numerical results are in good agreement with theoretical findings by Carleson [19]. 


\section{The Laplacian in Stretched Spherical Coordinates}

Let us introduce in the real space the usual spherical coordinate system:

$$
\left\{\begin{array}{l}
x=r \sin \vartheta \cos \varphi, \\
y=r \sin \vartheta \sin \varphi, \\
z=r \cos \vartheta,
\end{array}\right.
$$

and the polar equations:

$$
r=R^{ \pm}(\vartheta, \varphi),
$$

relevant to the boundaries of the supershaped shell $\mathcal{S}$ which is described by the following chain of inequalities:

$$
R^{-}(\vartheta, \varphi) \leq r \leq R^{+}(\vartheta, \varphi),
$$

with $0 \leq \vartheta \leq \pi$ and $0 \leq \varphi \leq 2 \pi$. In $(2), R^{ \pm}(\vartheta, \varphi)$ are assumed to be piece-wise $C^{2}$ functions satisfying the condition:

$$
R^{+}(\vartheta, \varphi)>R^{-}(\vartheta, \varphi)>0,0 \leq \vartheta \leq \pi, 0 \leq \varphi \leq 2 \pi .
$$

In this way, upon introducing the stretched radius $\rho$ such that:

$$
r=\frac{(b-\rho) R^{-}(\vartheta, \varphi)-(a-\rho) R^{+}(\vartheta, \varphi)}{b-a},
$$

with $b>a>0$, the considered shell-like domain $\mathcal{S}$ can be readily obtained by assuming $0 \leq \vartheta \leq \pi, 0 \leq \varphi \leq$ $2 \pi$ and $a \leq \rho \leq b$.

Remark: Note that, in the stretched coordinate system $\rho, \vartheta, \varphi$ the original domain $\mathcal{S}$ is transformed into the spherical shell of radii $a$ and $b$, respectively. Hence, in this system one can use classical techniques to solve the Helmholtz equation, including the eigenfunction method [1].

Let us consider a piece-wise $C^{2}(\stackrel{\circ}{\mathcal{S}})$ function $v(x, y$, $z)=v(r \sin \vartheta \cos \varphi, r \sin \vartheta \sin \varphi, r \cos \vartheta)=u(r, \vartheta, \varphi)$ and the Laplace operator, $\Delta$, in spherical coordinates:

$$
\begin{aligned}
\Delta u= & \frac{1}{r^{2}} \frac{\partial}{\partial r}\left(r^{2} \frac{\partial u}{\partial r}\right)+\frac{1}{r^{2} \sin \vartheta} \frac{\partial}{\partial \vartheta}\left(\sin \vartheta \frac{\partial u}{\partial \vartheta}\right) \\
& +\frac{1}{r^{2} \sin ^{2} \vartheta} \frac{\partial^{2} u}{\partial \varphi^{2}} .
\end{aligned}
$$

In the considered stretched coordinate system, $\Delta$ can be represented by setting:

$$
\begin{aligned}
& U(\rho, \vartheta, \varphi) \\
= & u\left(\frac{(b-\rho) R^{-}(\vartheta, \varphi)-(a-\rho) R^{+}(\vartheta, \varphi)}{b-a}, \vartheta\right) .
\end{aligned}
$$

In this way, by denoting $R^{ \pm}(\vartheta, \varphi)$ as $R^{ \pm}$for the sake of shortness, one can readily find:

$$
\begin{gathered}
\frac{\partial u}{\partial r}=\frac{b-a}{R^{+}-R^{-}} \frac{\partial U}{\partial \rho}, \\
\frac{\partial^{2} u}{\partial r^{2}}=\left(\frac{b-a}{R^{+}-R^{-}}\right)^{2} \frac{\partial^{2} U}{\partial \rho^{2}}, \\
\frac{\partial u}{\partial \vartheta}=-\frac{R_{\vartheta}}{R^{+}-R^{-}} \frac{\partial U}{\partial \rho}+\frac{\partial U}{\partial \vartheta},
\end{gathered}
$$

$$
\begin{aligned}
\frac{\partial^{2} u}{\partial \vartheta^{2}}= & \frac{2 R_{\vartheta}\left(R_{\vartheta}^{+}-R_{\vartheta}^{-}\right)-R_{\vartheta \vartheta}\left(R^{+}-R^{-}\right)}{\left(R^{+}-R^{-}\right)^{2}} \frac{\partial u}{\partial \rho} \\
& +\left(\frac{R_{\vartheta}}{R^{+}-R^{-}}\right)^{2} \frac{\partial^{2} U}{\partial \rho^{2}}-2 \frac{R_{\vartheta}}{R^{+}-R^{-}} \frac{\partial^{2} U}{\partial \rho \partial \vartheta}+\frac{\partial^{2} U}{\partial \vartheta^{2}} \\
\frac{\partial^{2} u}{\partial \varphi^{2}}= & \frac{2 R_{\varphi}\left(R_{\varphi}^{+}-R_{\varphi}^{-}\right)-R_{\varphi \varphi}\left(R^{+}-R^{-}\right)}{\left(R^{+}-R^{-}\right)^{2}} \frac{\partial U}{\partial \rho} \\
& +\left(\frac{R_{\varphi}}{R^{+}-R^{-}}\right)^{2} \frac{\partial^{2} U}{\partial \rho^{2}}-2 \frac{R_{\varphi}}{R^{+}-R^{-}} \frac{\partial^{2} U}{\partial \rho \partial \varphi}+\frac{\partial^{2} U}{\partial \varphi^{2}}
\end{aligned}
$$

with $R=(b-\rho) R^{-}-(a-\rho) R^{+}$, and where the subscripts denote the partial differentiation with respect to the polar angles $\vartheta$ and $\varphi$. Substituting Equations (8)(12) into Equation (6) finally yields Equation (13).

$$
\begin{aligned}
\Delta u & =\left(\frac{b-a}{R^{+}-R^{-}}\right)^{2}\left\{\left[1+\left(\frac{R_{\vartheta}}{R}\right)^{2}+\left(\frac{R_{\varphi}}{R \sin \vartheta}\right)^{2}\right] \frac{\partial^{2} U}{\partial \rho^{2}}\right. \\
& +\frac{R^{+}-R^{-}}{R}\left[2\left(1+\frac{R_{\vartheta}^{+}-R_{\vartheta}^{-}}{R^{+}-R^{-}} \frac{R_{\vartheta}}{R}+\frac{R_{\varphi}^{+}-R_{\varphi}^{-}}{R^{+}-R^{-}} \frac{R_{\varphi}}{R \sin ^{2} \vartheta}\right)-\frac{1}{R}\left(R_{\vartheta \vartheta}+R_{\vartheta} \cot \vartheta+\frac{R_{\varphi \varphi}}{\sin ^{2} \vartheta}\right)\right] \frac{\partial U}{\partial \rho} \\
& \left.+\left(\frac{R^{+}-R^{-}}{R}\right)^{2}\left(\frac{\partial^{2} U}{\partial \vartheta^{2}}+\cot \vartheta \frac{\partial U}{\partial \vartheta}+\frac{1}{\sin ^{2} \vartheta} \frac{\partial^{2} U}{\partial \varphi^{2}}\right)-2 \frac{R^{+}-R^{-}}{R^{2}}\left(R_{\vartheta} \frac{\partial^{2} U}{\partial \rho \partial \vartheta}+\frac{R_{\varphi}}{\sin ^{2} \vartheta} \frac{\partial^{2} U}{\partial \rho \partial \varphi}\right)\right\} .
\end{aligned}
$$


As it can be easily noticed, upon setting $R^{-}(\vartheta, \varphi)=$ $a=0$ and $R^{+}(\vartheta, \varphi)=b=1$, the classical expression of the Laplacian in spherical coordinates is recovered.

\section{The Robin Problem for the Helmholtz Equation}

Let us consider the interior Robin problem for the Helmholtz equation in a starlike shell $\mathcal{S}$, whose boundaries $\partial^{ \pm} \mathcal{S}$ are described by the polar equations $r=R^{ \pm}(\vartheta, \varphi)$ respectively:

$$
\left\{\begin{array}{l}
\Delta v(x, y, z)+k^{2} v(x, y, z)=0, \\
(x, y, z) \in \mathcal{S}, \\
\lambda^{ \pm} v(x, y, z)+\gamma^{ \pm} \frac{\partial v}{\partial v}(x, y, z)=f^{ \pm}(x, y, z), \\
(x, y, z) \in \partial^{ \pm} \mathcal{S},
\end{array}\right.
$$

where $k>0$ denotes the propagation constant, $\hat{v}^{ \pm}=$ $\hat{v}^{ \pm}(\vartheta, \varphi)$ are the outward-pointing normal unit vectors to the domain boundaries $\partial^{ \pm} \mathcal{S}$, respectively, and $\lambda^{ \pm}$, $\gamma^{ \pm}$are given regular weighting coefficients.

Under the mentioned assumptions, one can prove the following theorem.

Theorem. Let:

$$
\begin{gathered}
\psi_{\vartheta}^{ \pm}(\vartheta, \varphi)=\frac{R_{\vartheta}^{ \pm}(\vartheta, \varphi)}{R^{ \pm}(\vartheta, \varphi)}, \\
\psi_{\phi}^{ \pm}(\vartheta, \varphi)=\frac{R_{\phi}^{ \pm}(\vartheta, \varphi)}{R^{ \pm}(\vartheta, \varphi) \sin \vartheta}, \\
\psi^{ \pm}(\vartheta, \varphi)=\sqrt{\psi_{\vartheta}^{ \pm}(\vartheta, \varphi)^{2}+\psi_{\phi}^{ \pm}(\vartheta, \varphi)^{2}},
\end{gathered}
$$

and

$$
\begin{aligned}
& f^{ \pm}\left(R^{ \pm}(\vartheta, \varphi) \sin \vartheta \cos \varphi,\right. \\
& \left.R^{ \pm}(\vartheta, \varphi) \sin \vartheta \sin \varphi, R^{ \pm}(\vartheta, \varphi) \cos \vartheta\right) \\
= & F^{ \pm}(\vartheta, \varphi) \\
= & \sum_{n=0}^{+\infty} \sum_{m=0}^{n} P_{n}^{m}(\cos \vartheta)\left(\alpha_{n, m}^{ \pm} \cos m \varphi+\beta_{n, m}^{ \pm} \sin m \varphi\right),
\end{aligned}
$$

where:

$$
\begin{aligned}
\left\{\begin{array}{c}
\alpha_{n, m}^{ \pm} \\
\beta_{n, m}^{ \pm}
\end{array}\right\}= & \epsilon_{m} \frac{2 n+1}{4 \pi} \frac{(n-m) !}{(n+m) !} \\
& \int_{0}^{2 \pi} \int_{0}^{\pi} F^{ \pm}(\vartheta, \varphi) P_{n}^{m}(\cos \vartheta)\left\{\begin{array}{c}
\cos m \varphi \\
\sin m \varphi
\end{array}\right\} \sin \vartheta \mathrm{d} \vartheta \mathrm{d} \varphi,
\end{aligned}
$$

$\epsilon_{m}$ being the usual Neumann's symbol and $P_{n}^{m}(\cdot)$ the associated Legendre function of the first kind and orders $n, m$. Then, the boundary-value problem (14) for the Helmholtz equation admits a classical solution $v(x, y, z)$ $\in L^{2}(\mathcal{S})$ such that the series expansion (20) holds true. In Equation (20) $h_{n}^{(\ell)}(z)=\sqrt{\frac{1}{2} \pi / z} H_{n+1 / 2}^{(\ell)}(z)$ denotes the spherical Hankel function of kind $\ell=1,2$ and order $n$. For each pair of indices $n \in \mathbb{N}_{0}:=\mathbb{N} \bigcup\{0\}$ and $m=0,1, \cdots, n$ introduce the terms reported in Equation (21), where:

$$
\dot{h}_{n}^{(\ell)}(z)=\frac{\mathrm{d}}{\mathrm{d} z} h_{n}^{(\ell)}(z)=-h_{n+1}^{(\ell)}(z)+\frac{n}{z} h_{n}^{(\ell)}(z),
$$

and

$$
\dot{P}_{n}^{m}(z)=\frac{\mathrm{d}}{\mathrm{d} z} P_{n}^{m}(z)=\frac{n z P_{n}^{m}(z)-(n+m) P_{n-1}^{m}(z)}{z^{2}-1} .
$$

Therefore, the coefficients $A_{\ell, n, m}, B_{\ell, n, m}$ in (20) can be determined by solving the infinite linear system:

$$
\begin{aligned}
& v\left(\frac{R(\rho, \vartheta, \varphi)}{b-a} \sin \vartheta \cos \varphi, \frac{R(\rho, \vartheta, \varphi)}{b-a} \sin \vartheta \sin \varphi, \frac{R(\rho, \vartheta, \varphi)}{b-a} \cos \vartheta\right) \\
&= U(\rho, \vartheta, \varphi) \\
&= \sum_{n=0}^{+\infty} \sum_{m=0}^{n} P_{n}^{m}(\cos \vartheta)\left[h_{n}^{(1)}\left(k \frac{R(\rho, \vartheta, \varphi)}{b-a}\right)\left(A_{1, n, m} \cos m \varphi+B_{1, n, m} \varphi\right)+h_{n}^{(2)}\left(k \frac{R(\rho, \vartheta, \varphi)}{b-a}\right)\left(A_{2, n, m} \cos m \varphi+B_{2, n, m} \sin m \varphi\right)\right], \\
& \times\left[\begin{array}{l}
\xi_{\ell, n, m}(\vartheta, \varphi) \\
\eta_{\ell, n, m}(\vartheta, \varphi)
\end{array}\right]=h_{n}^{(\ell)}\left(k R^{ \pm}(\vartheta, \varphi)\right) P_{n}^{m}(\cos \vartheta) \\
&\left.\lambda^{ \pm}\left[\begin{array}{l}
\cos m \varphi \\
\sin m \varphi
\end{array}\right] \pm \frac{\gamma^{ \pm}}{\sqrt{1+\psi^{ \pm}(\vartheta, \varphi)^{2}}} \cdot\left[\begin{array}{cc}
\cos m \varphi & -\sin m \varphi \\
\sin m \varphi & \cos m \varphi
\end{array}\right] \cdot\left[\begin{array}{c}
\left.\left.k \frac{\dot{h}_{n}^{(\ell)}\left(k R^{ \pm}(\vartheta, \varphi)\right)}{h_{n}^{(\ell)}\left(k R^{ \pm}(\vartheta, \varphi)\right)}+\sin \vartheta \frac{\psi_{\vartheta}^{ \pm}(\vartheta, \varphi)}{R^{ \pm}(\vartheta, \varphi)} \frac{\dot{P}_{n}^{m}(\cos \vartheta)}{P_{n}^{m}(\cos \vartheta)}\right]\right\}, \\
-\frac{m}{\sin \vartheta} \frac{\psi_{\phi}^{ \pm}(\vartheta, \varphi)}{R^{ \pm}(\vartheta, \varphi)}
\end{array}\right]\right\},
\end{aligned}
$$




$$
\sum_{n=0}^{+\infty} \sum_{m=0}^{n}\left[\begin{array}{cccc}
X_{1,1, p, q, n, m}^{-} & Y_{1,1, p, q, n, m}^{-} & X_{1,2, p, q, n, m}^{-} & Y_{1,2, p, q, n, m}^{-} \\
X_{2,1, p, q, n, m}^{-} & Y_{2,1, p, q, n, m}^{-} & X_{2,2, p, q, n, m}^{-} & Y_{2,2, p, q, n, m}^{-} \\
X_{1,1, p, q, n, m}^{+} & Y_{1,1, p, q, n, m}^{+} & X_{1,2, p, q, n, m}^{+} & Y_{1,2, p, q, n, m}^{+} \\
X_{2,1, p, q, n, m}^{+} & Y_{2,1, p, q, n, m}^{+} & X_{2,2, p, q, n, m}^{+} & Y_{2,2, p, q, n, m}^{+}
\end{array}\right] \cdot\left[\begin{array}{c}
A_{1, n, m} \\
B_{1, n, m} \\
A_{2, n, m} \\
B_{2, n, m}
\end{array}\right]=\left[\begin{array}{c}
\alpha_{p, q}^{-} \\
\beta_{p, q}^{-} \\
\alpha_{p, q}^{+} \\
\beta_{p, q}^{+}
\end{array}\right],
$$

where:

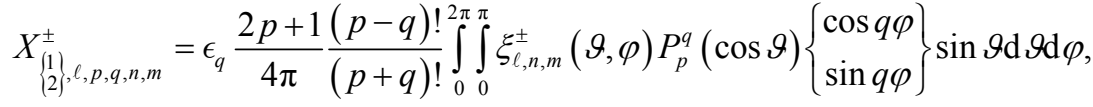

$$
\begin{aligned}
& Y_{\left\{\begin{array}{l}
\{1 \\
2\}
\end{array}\right\},, p, q, n, m}^{ \pm}=\epsilon_{q} \frac{2 p+1}{4 \pi} \frac{(p-q) !}{(p+q) !} \int_{0}^{2 \pi} \int_{0}^{\pi} \eta_{\ell, n, m}^{ \pm}(\vartheta, \varphi) P_{p}^{q}(\cos \vartheta)\left\{\begin{array}{c}
\cos q \varphi \\
\sin q \varphi
\end{array}\right\} \sin \vartheta \mathrm{d} \vartheta \mathrm{d} \varphi,
\end{aligned}
$$

with $\ell=1,2$ and $p \in \mathbb{N}_{0}, q=0,1, \cdots, p$.

Proof: Upon noting that in the stretched coordinate system $\rho, \vartheta, \varphi$ introduced in the $x, y, z$ space, the considered domain $\mathcal{S}$ turns into the spherical shell of radii $a$ and $b$, one can readily adopt the usual eigenfunction method [1] in combination with the separation of variables (with respect to $r, \vartheta$ and $\varphi$ ). As a consequence, elementary solutions of the problem can be searched in the form:

$$
\begin{aligned}
& u(r, \vartheta, \varphi) \\
= & U\left(\frac{b\left[r-R^{-}(\vartheta, \varphi)\right]-a\left[r-R^{+}(\vartheta, \varphi)\right]}{R^{+}(\vartheta, \varphi)-R^{-}(\vartheta, \varphi)}, \vartheta, \varphi\right) \\
= & P(\rho) \Theta(\vartheta) \Phi(\varphi) .
\end{aligned}
$$

Substituting into the Helmholtz equation, one easily finds that the functions $P(\cdot), \Theta(\cdot)$, and $\Phi(\cdot)$ must satisfy the ordinary differential equations:

$$
\begin{gathered}
r^{2} \frac{\mathrm{d}^{2} P(r)}{\mathrm{d} r^{2}}+2 r \frac{\mathrm{d} P(r)}{\mathrm{d} r}+\left[(k r)^{2}-v^{2}\right] P(r)=0, \\
\frac{1}{\sin \vartheta} \frac{\mathrm{d}}{\mathrm{d} \vartheta}\left[\sin \vartheta \frac{\mathrm{d} \Theta(\vartheta)}{\mathrm{d} \vartheta}\right]+\left(v^{2}-\frac{\mu^{2}}{\sin ^{2} \vartheta}\right) \Theta(\vartheta)=0 \\
\frac{\mathrm{d}^{2} \Phi(\varphi)}{\mathrm{d} \varphi^{2}}+\mu^{2} \Phi(\varphi)=0
\end{gathered}
$$

respectively. The parameters $v$ and $\mu$ are separation constants, whose choice is governed by the physical requirement that at any fixed point in space the scalar field $u(r, \vartheta, \varphi)$ must be single-valued. So, by setting:

$$
\begin{gathered}
\mu=m \in \mathbb{Z}, \\
v^{2}=n(n+1)\left(n \in \mathbb{N}_{0}\right),
\end{gathered}
$$

one can easily find:

$$
\begin{gathered}
\Phi(\varphi)=a_{m} \cos m \varphi+b_{m} \sin m \varphi, \\
\Theta(\vartheta)=c_{n, m} P_{n}^{m}(\cos \vartheta),
\end{gathered}
$$

where $a_{m}, b_{m}, c_{n, m} \in \mathbb{C}$ denote arbitrary constants. In order to identify the radial function $P(\cdot)$ introduced in (27), it is convenient to set:

$$
P(r)=(k r)^{-\frac{1}{2}} \zeta(r) .
$$

In this way, it is readily shown that $\zeta(r)$ satisfies:

$$
r^{2} \frac{\mathrm{d}^{2} \zeta(r)}{\mathrm{d} r^{2}}+r \frac{\mathrm{d} \zeta(r)}{\mathrm{d} r}+\left[(k r)^{2}-\left(n+\frac{1}{2}\right)^{2}\right] \zeta(r)=0,
$$

and, hence, is a cylinder function of half order that, without loss of generality, can be expressed as a linear combination of ordinary Hankel functions of first and second kind must, so that:

$$
P(r)=d_{n} h_{n}^{(1)}(k r)+e_{n} h_{n}^{(2)}(k r),
$$

with $d_{n}, e_{n} \in \mathbb{C}$. Therefore, the general solution of the Robin problem (14) can be searched in the form:

$$
\begin{aligned}
& u(r, \vartheta, \varphi) \\
&=\sum_{n=0}^{+\infty} \sum_{m=0}^{n} P_{n}^{m}(\cos \vartheta)\left[h_{n}^{1}(k r)\left(A_{1, n, m} \cos m \varphi+B_{1, n, m} \sin m \varphi\right)\right. \\
&\left.+h_{n}^{2}(k r)\left(A_{2, n, m} \cos m \varphi+B_{2, n, m} \sin m \varphi\right)\right] .
\end{aligned}
$$

Enforcing the Robin boundary condition yields:

$$
\begin{aligned}
& F^{ \pm}(\vartheta, \varphi) \\
= & \lambda^{ \pm} u\left(R^{ \pm}(\vartheta, \varphi), \vartheta, \varphi\right)+\gamma^{ \pm} \frac{\partial u}{\partial v}\left(R^{ \pm}(\vartheta, \varphi), \vartheta, \varphi\right) \\
= & \lambda^{ \pm} u\left(R^{ \pm}(\vartheta, \varphi), \vartheta, \varphi\right)+\gamma^{ \pm} \nabla u\left(R^{ \pm}(\vartheta, \varphi), \vartheta, \varphi\right) \cdot \hat{v}^{ \pm}(\vartheta, \varphi),
\end{aligned}
$$

where:

$$
\begin{aligned}
\nabla u(r, \vartheta, \varphi)= & \hat{r} \frac{\partial u(r, \vartheta, \varphi)}{\partial r}+\hat{\vartheta} \frac{1}{r} \frac{\partial u(r, \vartheta, \varphi)}{\partial \vartheta} \\
& +\hat{\varphi} \frac{1}{r \sin \vartheta} \frac{\partial u(r, \vartheta, \varphi)}{\partial \varphi},
\end{aligned}
$$


and:

$$
\hat{v}^{ \pm}(\vartheta, \varphi)= \pm \frac{\hat{r}-\psi_{\vartheta}^{ \pm}(\vartheta, \varphi) \hat{\vartheta}-\psi_{\phi}^{ \pm}(\vartheta, \varphi) \hat{\varphi}}{\sqrt{1+\psi^{ \pm}(\vartheta, \varphi)^{2}}} .
$$

Hence, combining equations above and using a classical harmonic projection method, the Equations (21)-(26) follow after some algebraic manipulations.

It is worth noting that the derived expressions still hold under the assumption that $R^{ \pm}(\vartheta, \varphi)$ are piecewise continuous functions, and the boundary values are described by square integrable, not necessarily continuous, functions, so that the relevant spherical harmonic coefficients $\alpha_{n, m}^{ \pm}, \beta_{n, m}^{ \pm}$in Equation (19) are finite quantities.

\section{Numerical Procedure}

In the following numerical examples, let us assume, for the boundaries $\partial^{ \pm} \mathcal{S}$ of the considered annulus, general polar equations of the type:

$$
\begin{aligned}
& R^{ \pm}(\vartheta, \varphi) \\
= & \left(\left|\frac{1}{d_{x}^{ \pm}} \sin \frac{a_{x}^{ \pm} \vartheta}{2} \cos \frac{b_{x}^{ \pm} \varphi}{4}\right|^{v_{x}^{ \pm}}\right. \\
& \left.+\left|\frac{1}{d_{y}^{ \pm}} \sin \frac{a_{y}^{ \pm} \vartheta}{2} \sin \frac{b_{y}^{ \pm} \varphi}{4}\right|^{\nu_{y}^{ \pm}}+\left|\frac{1}{d_{z}^{ \pm}} \cos \frac{a_{z}^{ \pm} \vartheta}{2}\right|^{\nu_{z}^{ \pm}}\right)^{-1 / v_{0}^{ \pm}},
\end{aligned}
$$

which provides an extension, to the three-dimensional case, of the "superformula" introduced by Gielis in [18] . Very different characteristic geometries, including ellipsoids, ovaloids, and Lamé-type domains (also called "superellipsoids") can be obtained by assuming suitable values of the parameters $a_{x}^{ \pm}, a_{y}^{ \pm}, a_{z}^{ \pm}, b_{x}^{ \pm}, b_{y}^{ \pm}, d_{x}^{ \pm}, d_{y}^{ \pm}, d_{z}^{ \pm}, v_{x}^{ \pm}$, $v_{y}^{ \pm}, v_{z}^{ \pm}, v_{0}^{ \pm}$in (42). It is to emphasized that almost all three-dimensional normal- polar shell-like domains can be described, or closely approximated, by the considered formula.

In order to assess the performance of the proposed methodology in terms of numerical accuracy and convergence rate, the relative boundary error has been evaluated according to Equation (43), where $\|\cdot\|$ is the usual $L^{2}$ norm, and $U_{N}=U_{N}(\rho, \vartheta, \varphi)$ denotes the partial sum of order $N$ relevant to the spherical harmonic expansion representing the solution of the boundary-value problem for the Helmholtz equation (see Equation (44)).

Remark: It is to be noted that, where the boundary values exhibit a rapidly oscillating behavior, the number $N$ of terms in the spherical harmonic expansion approximating the solution of the problem should be increased accordingly in order to achieve the desired numerical accuracy.

\section{Example}

Upon assuming in (42) $a_{x}^{ \pm}=a_{y}^{ \pm}=a_{z}^{ \pm}=2, b_{x}^{ \pm}=b_{y}^{ \pm}=4$, $d_{x}^{-}=d_{y}^{-}=d_{z}^{-}=4 / 5, d_{x}^{+}=d_{y}^{+}=d_{z}^{+}=1, v_{x}^{ \pm}=v_{y}^{ \pm}=v_{z}^{ \pm}=3$, and $v_{0}^{ \pm}=2$, the shell $\mathcal{S}$ turns to feature a cuboidal shape. Let $f_{-}(x, y, z)=\sin 2 x+\cos 3 y+\mathrm{e}^{-2 y^{2}-3 z^{2}+1}$ and $f_{+}(x, y, z)=x^{3} y^{3} z^{3}+\mathrm{e}^{x+y+z}-x+2 y-3 z$ be the functions describing the boundary values. Provided that the propagation constant is $k=2 \pi$, and $\lambda_{-}=-2 / 5, \lambda_{+}=-1$, $\gamma_{-}=1 / 10, \gamma_{+}=-1 / 2$ are the weighting coefficients in the Robin condition, the relative boundary error $e_{N}$ as function of the number $N$ of terms in the truncated series expansion (44) exhibits the behavior shown in Figure 1. As it appears from Figure 2, the selection of the expansion order $N=11$ leads to a very accurate representation of the solution $v_{N}(x, y, z)$, whose spherical harmonic coefficients $A_{\ell, n, m}$ and $B_{\ell, n, m}(\ell=1,2)$ are plotted in Figure 3.

Remark: It has been observed that $L^{2}$ norm of the difference between the exact solution and the relevant approximation is generally negligible. Point-wise convergence seems to be verified in the considered domains, with the only exception of a set of measure zero consisting of quasi-cusped points. In the neighbourhood of these points, oscillations of the truncated order solution, recalling the classical Gibbs phenomenon, usually take place.

$$
\begin{array}{r}
e_{N}=\sqrt{\frac{\left\|\lambda^{-} U_{N}+\gamma^{-} \frac{\partial U_{N}}{\partial v}-F^{-}\right\|_{\partial^{-} \mathcal{S}}^{2}+\left\|\lambda^{+} U_{N}+\gamma^{+} \frac{\partial U_{N}}{\partial v}-F^{+}\right\|_{\partial^{+} \mathcal{S}}^{2}}{\left\|F^{-}\right\|_{\partial^{-} \mathcal{S}}^{2}+\left\|F^{+}\right\|_{\partial^{+} \mathcal{S}}^{2}}}, \\
U_{N}(\rho, \vartheta, \varphi)=\sum_{n=0}^{N} \sum_{m=0}^{n} P_{n}^{m}(\cos \vartheta)\left[h_{n}^{(1)}\left(k \frac{R(\rho, \vartheta, \varphi)}{b-a}\right)\left(A_{1, n, m} \cos m \varphi+B_{1, n, m} \sin m \varphi\right)\right. \\
\left.+h_{n}^{(2)}\left(k \frac{R(\rho, \vartheta, \varphi)}{b-a}\right)\left(A_{2, n, m} \cos m \varphi+B_{2, n, m} \sin m \varphi\right)\right] .
\end{array}
$$




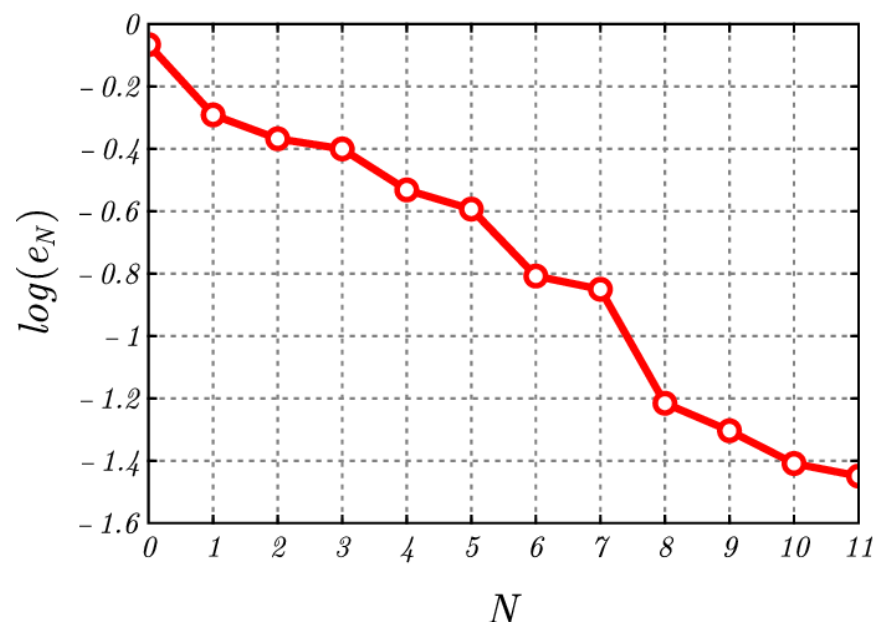

Figure 1. Relative boundary error $e_{N}$ as function of the order $N$ of the truncated spherical harmonic expansion representing the solution of the considered Robin problem for the Helmholtz equation in the supershaped shell $\mathcal{S}$ described by the generalized Gielis formula with parameters $a_{x}^{ \pm}=a_{y}^{ \pm}=a_{z}^{ \pm}=2, b_{x}^{ \pm}=b_{y}^{ \pm}=4, d_{x}^{-}=d_{y}^{-}=d_{z}^{-}=4 / 5, d_{x}^{+}=d_{y}^{+}=d_{z}^{+}=1, v_{x}^{ \pm}=v_{y}^{ \pm}=v_{z}^{ \pm}=3$,

$v_{0}^{ \pm}=2$.
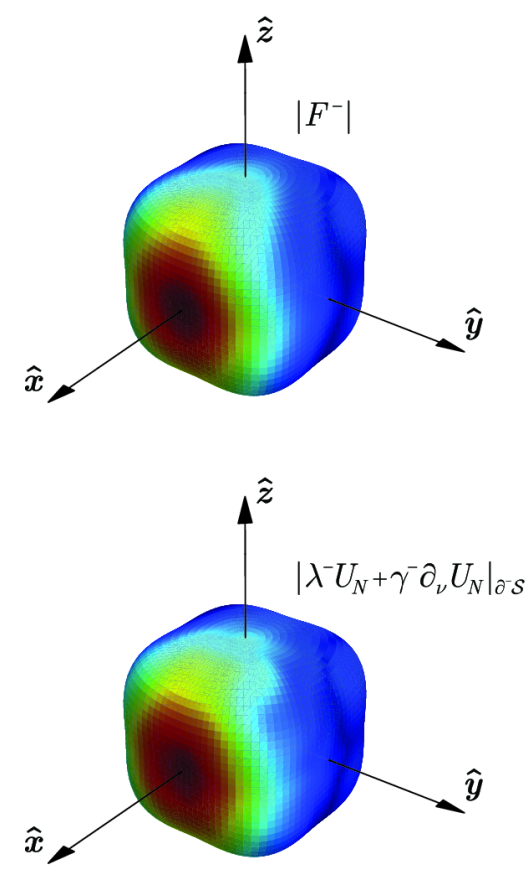

(a)

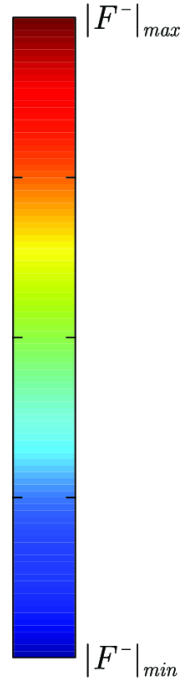

(b)

Figure 2. Boundary behavior along $\partial^{-} \mathcal{S}$ (a) and $\partial^{+} \mathcal{S}$ (b) of the partial sum $U_{N}(\rho, \vartheta, \varphi)$ of order $N=11$ representing the solution of the considered Robin problem for the Helmholtz equation in the supershaped shell $\mathcal{S}$.

\section{Conclusion}

A harmonic projection method, in combination with the adoption of a suitable stretched coordinate system, has been developed for solving the Robin problem for the Helmholtz equation in supershaped shell. In this way, analytically based expressions of the solution of the considered class of $B V P \mathrm{~s}$ can be derived by using classical

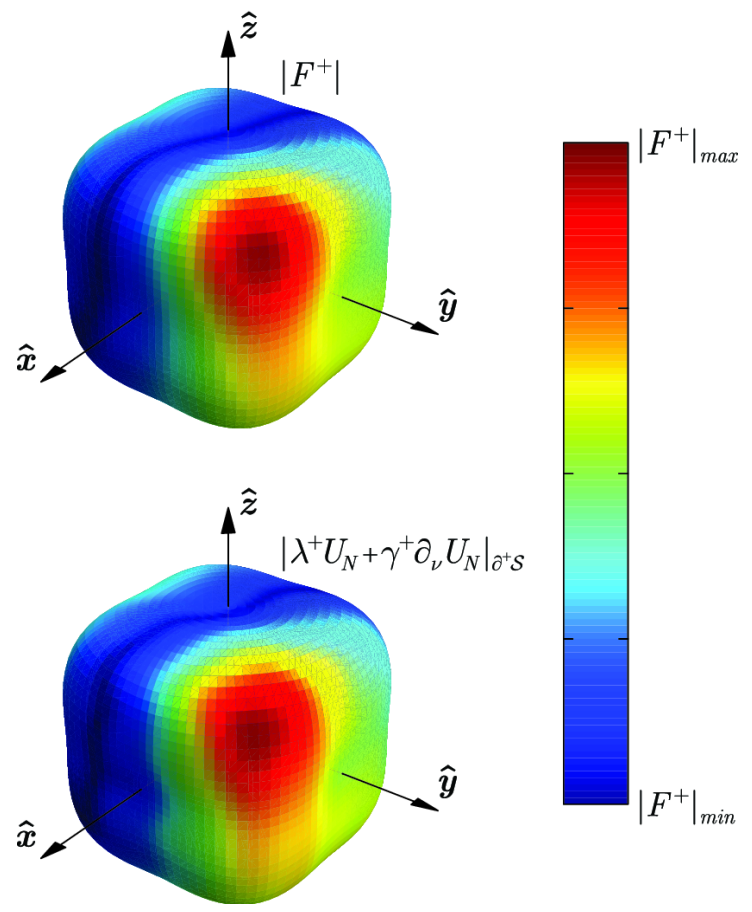
quadrature rules, so overcoming the need for cumbersome numerical techniques such as finite-difference or finite-element methods. The proposed approach has been successfully validated by means of a dedicated numerical procedure based on the computer-aided algebra tool Mathematica ${ }^{\circ}$. A point-wise convergence of the expansion series representing the solution seems to be verified 


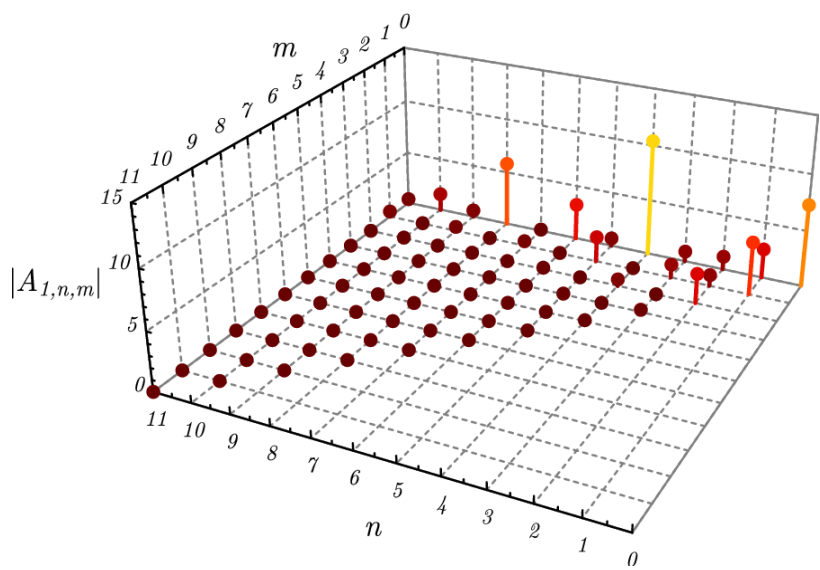

(a)

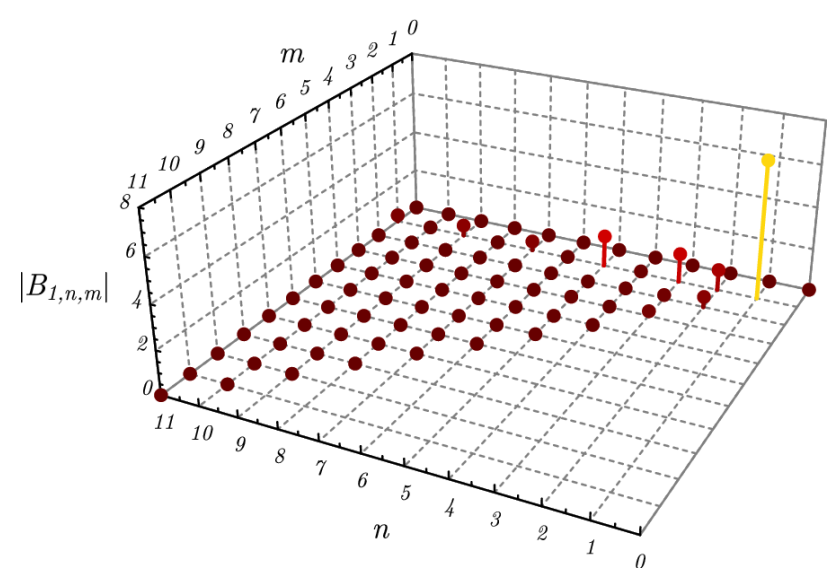

(b)

Figure 3. Magnitude of the coefficients $A_{\ell, n, m}$ and $B_{\ell, n, m}(\ell=1,2)$ relevant to the spherical harmonic expansion $v_{N}(x, y, z)$ $(N=11)$ representing the solution of the considered Robin problem for the Helmholtz equation in the supershaped shell $\mathcal{S}$.

with the only exception of a set of measure zero consisting of the quasi-cusped points along the boundary of the problem domain. In these points, Gibbs-like oscillations may occur. The computed results are found to be in good agreement with the theoretical findings on Fourier series.

\section{REFERENCES}

[1] N. N. Lebedev, "Special Functions and Their Applications," Dover Inc., New York, 1972.

[2] G. Krall, "Meccanica Tecnica Delle Vibrazioni," Vol. 2, Veschi, Roma, 1970.

[3] A. Bondeson, T. Rylander and P. Ingelstrom, "Computational Electromagnetics," Springer Science, New York, 2005.

[4] D. Medková, "Solution of the Dirichlet Problem for the Laplace Equation," Applications of Mathematics, Vol. 44, No. 2, 1999, pp. 143-168. doi:10.1023/A:1022209421576

[5] B. N. Khoromski, "Integro-Difference Method of Solution of the Dirichlet Problem for the Laplace Equation," Zhurnal Vychislitel'noi Matematiki i Matematicheskoi Fiziki, Vol. 24, No. 1, 1984, pp. 53-64.

[6] A. P. Volkov, "An Effective Method for Solving the Dirichlet Problem for the Laplace Equation," Differentsial'nye Uravneniya, Vol. 19, 1983, pp. 1000-1007.

[7] D. M. Young, "Iterative Methods for Solving Partial Difference Equations of Elliptic Type," Transactions on American Mathematical Society, Vol. 76, 1954, pp. 92111. doi:10.1090/S0002-9947-1954-0059635-7

[8] G. P. Tolstov, "Fourier Series," Dover Inc., New York, 1962.

[9] P. Natalini, R. Patrizi, and P. E. Ricci, "The Dirichlet Problem for the Laplace Equation in a Starlike Domain of a Riemann Surface," Numerical Algorithms, Vol. 28, No. 1-4, 2001, pp. 215-227. doi:10.1023/A:1014059219005

[10] D. Caratelli and P. E. Ricci, "The Dirichlet Problem for the Laplace Equation in a Starlike Domain," Proceedings of International Conference on Scientific Computing, Las Vegas, 14-17 July 2008, pp. 160-166.

[11] D. Caratelli, B. Germano, J. Gielis, M. X. He, P. Natalini and P. E. Ricci, "Fourier Solution of the Dirichlet Problem for the Laplace and Helmholtz Equations in Starlike Domains," Lecture Notes of Tbilisi International Centre of Mathematics and Informatics, Tbilisi University Press, Tbilisi, 2010.

[12] D. Caratelli, P. Natalini, P. E. Ricci and A. Yarovoy, "The Neumann Problem for the Helmholtz Equation in a Starlike Planar Domain," Applied Mathematics and Computation, Vol. 216, No. 2, 2010, pp. 556-564. doi:10.1016/i.amc.2010.01.077

[13] D. Caratelli, J. Gielis, P. Natalini, P. E. Ricci and I. Tavkelidze, "The Robin Problem for the Helmholtz Equation in a Starlike Planar Domain," Georgian Mathematical Journal, Vol. 18, No. 3, 2011, pp. 465-480.

[14] D. Caratelli, J. Gielis and P. E. Ricci, "Fourier-Like Solution of the Dirichlet Problem for the Laplace Equation in k-Type Gielis Domains," Journal of Pure and Applied Mathematics: Advances and Applications, Vol. 5, No. 2, 2011, pp. 99-111.

[15] D. Caratelli, P. E. Ricci and J. Gielis, "The Robin Problem for the Laplace Equation in a Three-Dimensional Starlike Domain," Applied Mathematics and Computation, Vol. 218, No. 3, 2011, pp. 713-719.

[16] J. Gielis, D. Caratelli, Y. Fougerolle, P. E. Ricci and T. Gerats, "Universal Natural Shapes from Unifying Shape Description to Simple Methods for Shape Analysis and Boundary Value Problems," PLoS One, Vol. 7, No. 9, 2012, Article ID: e29324. doi:10.1371/journal.pone.0029324

[17] G. Dattoli, B. Germano, M. R. Martinelli and P. E. Ricci, "A Novel Theory of Legendre Polynomials," Mathematical and Computer Modelling, Vol. 54, No. 1-2, 2011, pp. 80-87. doi:10.1016/j.mcm.2011.01.037

[18] J. Gielis, "A Generic Geometric Transformation that Unifies a Wide Range of Natural and Abstract Shapes," 
American Journal of Botany, Vol. 90, No. 3, 2003, pp. 333-338. doi:10.3732/ajb.90.3.333

[19] L. Carleson, "On Convergence and Growth of Partial
Sums of Fourier Series," Acta Mathematica, Vol. 116, No. 1, 1966, pp. 135-157. doi:10.1007/BF02392815 